$\mathrm{e}^{+} \mathrm{e}^{-}$Collisions from Phi to Psi 2013 (PHIPSI2013)

International Journal of Modern Physics: Conference Series

Vol. 35 (2014) 1460438 (6 pages)

(C) The Author

DOI: $10.1142 / \mathrm{S} 2010194514604384$

\title{
HIGH ACCURATE MEASUREMENT OF $\tau$ MASS AT BESIII
}

\author{
XIAOHU MO \\ on behalf of BESIII Collaboration \\ Institute of High Energy Physics, Chinese Academy of Sciences, \\ P.O. Box 918-1, Beijing 100049, China \\ moxh@ihep.ac.cn
}

Published 18 December 2014

\begin{abstract}
The new experiment at BESIII aims at the uncertainty less than $0.1 \mathrm{MeV}$, or $5.6 \times$ $10^{-5}$ for the relative error. To make full use of machine time, the optimization study is performed to acquire the best strategy of data taking. The results of $m_{\tau}$ based on the test scan sample at BESIII are presented.
\end{abstract}

Keywords: $\tau$ mass; scan optimization; uncertainty.

PACS Numbers: 14.60.Fg, 02.60.Pn, 02.70.Uu

\section{Introduction}

Improvement of accuracy of $\tau$ mass $\left(m_{\tau}\right)$ is needed in its own right. Listed as follows are the measured mass values of three leptons according to PDG2012: ${ }^{1}$

$$
\begin{aligned}
& m_{e}=0.510998910 \pm 0.000000013 \mathrm{MeV} \quad\left(\delta m_{e} / m_{e} \approx 2.554 \times 10^{-8}\right), \\
& m_{\mu}=105.658367 \pm 0.000004 \mathrm{MeV} \quad\left(\delta m_{\mu} / m_{\mu} \approx 3.786 \times 10^{-8}\right), \\
& m_{\tau}=1776.82 \pm 0.16 \mathrm{MeV} \quad\left(\delta m_{\tau} / m_{\tau} \approx 9.568 \times 10^{-5}\right) .
\end{aligned}
$$

As one of three elementary leptons in nature, the accuracy of $m_{\tau}$ is almost 4 orders of magnitude lower than the other two leptons, that is electron and muon, whose accuracy is already at the level of $10^{-8}$.

One well-known motivation for getting accurate $m_{\tau}$ consists in the lepton universality test. By the light of the following formula

$$
\left(\frac{g_{\tau}}{g_{\mu}}\right)^{2}=\left(\frac{m_{\mu}}{m_{\tau}}\right)^{5} \frac{\tau_{\mu}}{\tau_{\tau}} \frac{B_{\tau \rightarrow e \nu \bar{\nu}}}{B_{\mu \rightarrow e \nu \bar{\nu}}}\left(1+\Delta_{e}\right),
$$

where $g_{\tau}$ and $g_{\mu}$ are $\tau$ - and $\mu$-related coupling constants; $\tau_{\tau}$ and $\tau_{\mu}, B_{\tau \rightarrow e \nu \bar{\nu}}$ and $B_{\mu \rightarrow e \nu \bar{\nu}}$ are the corresponding life times and decay branching ratios, respectively;

This is an Open Access article published by World Scientific Publishing Company. It is distributed under the terms of the Creative Commons Attribution 3.0 (CC-BY) License. Further distribution of this work is permitted, provided the original work is properly cited. 
$\Delta_{e}$ is the correct factor (which in fact includes the contributions from phase factor, radiative correction factor of $\mathrm{QED}$, correct factor of propagator of $\mathrm{W}$-meson etc.), the universality test is sensitive to $m_{\tau}$ because of 5 power dependence.

Another test also depends only on the accuracy of $m_{\tau}$. Here is an interesting formula of masses of three leptons discovered in 1981: ${ }^{2}$

$$
m_{e}+m_{\mu}+m_{\tau}=\frac{2}{3}\left(\sqrt{m_{e}}+\sqrt{m_{\mu}}+\sqrt{m_{\tau}}\right)^{2} .
$$

According to the error propagation formula $\left(f_{m}\right.$ indicates the difference between the right and left sides of Eq. (3))

$$
\delta f_{m}=\sqrt{\sum_{k=e, \mu, \tau}\left[m_{k}-\frac{2}{3}\left(\sum_{i=e, \mu, \tau} \sqrt{m_{k} m_{i}}\right)\right]^{2} \cdot\left(\frac{\delta m_{k}}{m_{k}}\right)^{2}} \approx \frac{1}{3} \delta m_{\tau},
$$

which indicates that the test of Eq. (3) depends almost merely on the accuracy of $m_{\tau}$.

Table 1. Measurement results of $m_{\tau}$ from different experiment groups.

\begin{tabular}{lllll}
\hline $\begin{array}{l}\text { Measured } m_{\tau} \\
\text { (value+statistic+systematic) }\end{array}$ & Year & Exp. Group & Data sample & Method \\
\hline $1776.68 \pm 0.12 \pm 0.41$ & 2009 & Babar $^{10}$ & $423 \mathrm{fb}^{-1}$ & Pseudo-mass \\
$1776.81_{-0.23}^{+0.25} \pm 0.15$ & 2007 & KEDR $^{9}$ & $6.7 \mathrm{fb}^{-1}$ & Threshold-scan \\
$1776.61 \pm 0.13 \pm 0.35$ & 2007 & Belle $^{8}$ & $414 \mathrm{fb}^{-1}$ & Pseudo-mass \\
$1776.96_{-0.21-0.17}^{+0.18+0.25}$ & 1996 & BES $^{5}$ & $5.1 \mathrm{fb}^{-1}$ & Threshold-scan \\
\hline
\end{tabular}

As a matter of fact, since the discovery of $\tau$ lepton, many experiments have been performed to measure $m_{\tau} \cdot{ }^{3-10}$ However, the value of $m_{\tau}$ in PDG2012 is based mostly on the four results as summarized in Table 1, where two results were acquired using the method of pseudo-mass while the others using the method of threshold scan. The latter method was and will be adopted by BES collaboration. The great improvement for the new experiment at BESIII is the establishment of the high accurate beam energy measurement system (BEMS) ${ }^{11}$ that decreases the systematic uncertainty due to beam energy to the level of $10^{-5}$. With this system and the expectable large data sample, it is time for BES to perform $\tau$ mass scan to get a good results.

\section{Mass measurement through scan}

For $m_{\tau}$ scan experiment, several points (say $N_{p t}$ points) need to be taken in the vicinity of $\tau$-pair threshold. The following likelihood function is constructed: ${ }^{5}$

$$
L F=\prod_{i}^{N_{p t}} \frac{\mu_{i}^{N_{i}} e^{-\mu_{i}}}{N_{i} !}
$$


where $N_{i}$ is the observed number of $\tau^{+} \tau^{-}$events obtained by $e \mu$-tagged final state ${ }^{\text {a }}$ at $i$-th scan point. Here $N_{i}$ is assumed obeying Poisson distribution, whose expectation $\mu_{i}$ is given by

$$
\mu_{i}\left(m_{\tau}\right)=\left[\epsilon \cdot B_{e \mu} \cdot \sigma_{o b s}\left(m_{\tau}, E_{c m}^{i}\right)+\sigma_{B G}\right] \cdot \mathcal{L}_{i}
$$

In above equation, $\mathcal{L}_{i}$ is the integrated luminosity at the point $i ; \epsilon$ is the overall efficiency of $e \mu$ final state for identifying $\tau^{+} \tau^{-}$events, which includes trigger efficiency and event selection efficiency; $B_{e \mu}$ is the combined branching ratio for decays $\tau^{+} \rightarrow e^{+} \nu_{e} \bar{\nu}_{\tau}$ and $\tau^{-} \rightarrow \mu^{-} \bar{\nu}_{\mu} \nu_{\tau}$, or the corresponding charge conjugate mode; $\sigma_{o b s}$ (with $m_{\tau}$ as a parameter), which can be calculated by the improved Voloshin's formulas, ${ }^{12}$ is the observed cross section measured at point $i$ with energy $E_{c m}^{i}$; and $\sigma_{B G}$ is the total cross section of background channels after $\tau^{+} \tau^{-}$selection. If $m_{\tau}$ is set as a free parameter, the minimization of $L F$ in Eq. (5) yields the best estimation for $m_{\tau}$.

The aim of the study of statistical optimization is to find out the scheme that can provide the highest precision on $m_{\tau}$ for a specified period of data taking time or equivalently for a given integrated luminosity. The main conclusions for the optimization study are summarized as follows: ${ }^{13-16}$

(1) For $N$ free parameters, $N$ scan points are enough;

(2) The optimal position can be obtained by single parameter scan;

(3) Luminosity allocation can be determined analytically or by simulation method;

(4) The uncertainty of $m_{\tau}$ is proportional to the inverse of square root of luminosity.

By virtue of these conclusions, the optimal scan strategy is made: firstly, $J / \psi$ scan (points 1-7), then scan in vicinity of $\tau$-pair threshold (points 8-12), last $\psi^{\prime}$ scan (points 13-19). After that, repeat (in $\tau$-region, the data are taken only at points 9 and 10). The two-circle process is designed to understand the stability of accelerator and detector. The key issue here is to acquire $100 \mathrm{pb}^{-1}$ data in $\tau$-region to guarantee the uncertainty of $m_{\tau}$ less than $0.1 \mathrm{MeV}$. The scan plan and part of offline results are summarized in Table 2.

\section{Data analysis}

At BEPCII, the electron's and positron's beams collide with an angle 22 mrad. Therefore, the center-of-mass energy $\left(E_{c m}\right)$ must be calculated with care. Notice $\alpha=22 \mathrm{mrad}$ is an small quantity, with conditions $E_{+,-} \gg m_{e}$ and $E_{+,-} \simeq E_{\text {beam }}$ $\left(E_{+,-}\right.$denote the beam energy $E_{\text {beam }}$ for positron $E_{+}$or electron $\left.E_{-}\right)$, it ready to obtain the formula

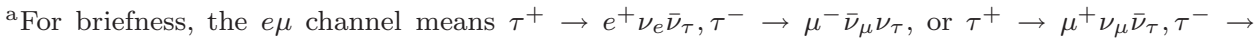
$e^{-} \bar{\nu}_{e} \nu_{\tau}$.
} 
Table 2. Scan plan and offline results. Superscript "exp" indicates the expected value of scan plan; while "offline" denotes the measured value from offline analysis. $E_{\text {beam }}$ and $\mathcal{L}$ represent the beam energy and integrated luminosity respectively. ( $\dagger$ : the expected values are listed in last two columns; $\ddagger$ : the measured values are little different from the expected ones due to the fluctuation of accelerator.)

\begin{tabular}{ccccccccc}
\hline $\begin{array}{c}\text { Energy } \\
\text { Region }\end{array}$ & order & $\begin{array}{c}E_{\text {beam }}^{\text {exp }} \\
(\mathrm{MeV})\end{array}$ & $\begin{array}{c}\mathcal{L}^{\text {exp }} \\
\left(\mathrm{pb}^{-1}\right)\end{array}$ & $\begin{array}{c}E_{\text {beam }}^{\text {offline }} \\
(\mathrm{MeV})\end{array}$ & $\begin{array}{c}\mathcal{L}^{\text {offline }} \\
\left(\mathrm{pb}^{-1}\right)\end{array}$ & \multicolumn{3}{c}{$E_{\text {beam }}^{\text {exp }}(\mathrm{MeV})$} \\
\hline$J / \psi$ & $1-7$ & $\dagger$ & 9 & $\ddagger$ & 1.51 & $1 / 13$ & 1544.0 & 1838.0 \\
\hline$\tau$ & 8 & 1771.0 & 14 & 1771.2 & 4.25 & $2 / 14$ & 1547.8 & 1841.9 \\
& 9 & 1776.6 & $14+25$ & 1776.9 & 5.57 & $3 / 15$ & 1548.2 & 1842.5 \\
& 10 & 1777.0 & $14+12$ & & & $4 / 16$ & 1548.6 & 1843.1 \\
& 11 & 1780.4 & 7 & 1780.6 & 3.89 & $5 / 17$ & 1549.0 & 1843.8 \\
& 12 & 1792.0 & 14 & 1800.1 & 9.55 & $6 / 18$ & 1549.4 & 1844.5 \\
\cline { 1 - 2 } & $13-19$ & $\dagger$ & 10 & $\ddagger$ & 7.53 & $7 / 19$ & 1552.0 & 1847.0 \\
\hline$\psi^{\prime}$ & & & & & & & & \\
\hline
\end{tabular}

$$
E_{c m} \simeq 2 E_{\text {beam }}\left(1-\frac{\alpha^{2}}{8}\right) .
$$

The effect due to the colliding angle is around $0.11 \mathrm{MeV}$, a very small quantity but can not be neglected for our accuracy requirement.

Since BESIII detector located at the south interaction point while BEMS located at the north crossing point, the data taking of two systems is independent on some extend, the matching of two kinds of information is fairly important, especially when the running status of accelerator or detector is not very stable. A great effects have been made to fix the values of $E_{c m}$ for resonance scans of $J / \psi$ and $\psi^{\prime}$.

Di-gamma and Bhabha events are adopted to calculate the luminosity, together with the number of selected hadron events, the cross section of resonance can be evaluated. Then, the fit of resonance parameters, especially resonance mass and energy spread, are performed. The corresponding results are used for the uncertainty estimation of energy calibration and energy spread determination.

For $\tau$-pair event selection, optimization for each criterion selection is performed. Totally thirteen channels are analyzed, that is $e e, e \mu, e \pi, e K, \mu \mu, \mu \pi, \mu K, \pi \pi, \pi K$, $K K, e \rho, \mu \rho$, and $\pi \rho$. Due to cut and energy difference, the efficiencies vary from $10 \%$ to $40 \%$. The comparison between data and Monte Carlo simulation reveals the good consistency.

The optimized fitting curve is shown in Fig. 1. The consistency between measured points and theoretical curve is up go the state of the art. Many systematic uncertainties have been studied, include the accuracy of theoretical formula, errors due to energy scale and energy spread, effect of efficiency and luminosity, and so on (refer to the table in Fig. 1 for the special values). The synthetic error is around 120 $\mathrm{keV}$, which is at the same level of the statistic error, and the total error is around $0.171 \mathrm{MeV}$ (or the relative error at the level of $9.6 \times 10^{-5}$ ). The final result is as follows:

$$
m_{\tau}=1776.82 \pm 0.12 \text { (stat.) } \pm 0.12 \text { (sys.) } \mathrm{MeV} \text {. }
$$




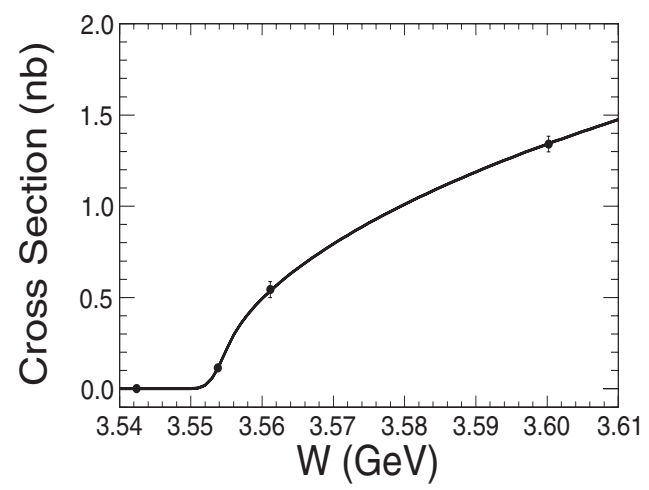

\begin{tabular}{lc}
\hline \multicolumn{2}{c}{ Systematic errors } \\
\hline Source & $\mathrm{keV}$ \\
\hline Theo. & 10 \\
ESpread $_{\text {Spale }}$ & 12 \\
$E_{\text {Scal }}$ & 90 \\
Evt.selection & 50 \\
Eff. & 48 \\
Bg.shape & 40 \\
Lum. & 6 \\
\hline Sum & 122 \\
\hline
\end{tabular}

Fig. 1. $\tau$ mass fit considering the effects due to the initial state radiation and the energy spread of accelerator.

\section{Summary}

Using around $20 \mathrm{pb}^{-1} \tau$ data, BESIII collaboration has measured the mass of $\tau$ lepton, whose central value is similar to that of PDG, while the uncertainty is comparable with that of PDG; Lepton universality is tested at the level of 5 per mille, and the mass equality of three leptons is established within the error of 57 $\mathrm{keV}$; Experience is acquired from the present data analysis, more detailed plan is made for the finer $m_{\tau}$ scan in the future.

\section{Acknowledgments}

This work is supported in part by the National Natural Science Foundation of China under Contracts Nos. 11375206, 11235011, Y21134005C, 10825524.

\section{References}

1. J. Beringer et al. (Particle Data Group), Phys. Rev. D 86, 010001 (2012).

2. Y. Koide, Phys. Rev. D 28, 252 (1983); Mod. Phys. Lett. A 5, 2319 (1990).

3. W. Bacino et al., Phys. Rev. Lett. 41, 13 (1978).

4. ARGUS Collab. (H. Albrecht et al.), Phys. Lett. B 292, 221 (1992).

5. BES Collab. (J.Z. Bai et al.), Phys. Rev. Lett. 69, 3021 (1992); Phys. Rev. D 53, 20 (1996).

6. CLEO Collab. (A. Anastassov et al.), Phys. Rev. D 55, 2559 (1997).

7. OPAL Collab. (G. Abbiendi et al.), Phys. Lett. B 492, 23 (2000).

8. Belle Collab. (K. Belous et al.), Phys. Rev. Lett. 99, 011801 (2007).

9. KEDR Collab. (V.V. Anashin et al.), J. Exp. The. Phys. Lett. 85, 347 (2007).

10. Babar Collab. (B. Aubert et al.), Phys. Rev. D 80, 092005 (2009).

11. X.H. MO et al., China Phys. C 32, 995 (2008);

X.H. MO et al., China Phys. C 34, 912 (2010);

E.V. Abakumova et al., Nucl. Instrum. Methods A 659, 21 (2011).

12. M.B. Voloshin, Phys. Lett. B 556, 153 (2003). 
X. H. Mo

13. Y.K. WANG et al., Nucl. Instrum. Methods A 583, 479 (2007).

14. Y.K. WANG et al., China Phys. C 33, 501 (2009).

15. M.N. Achasov et al., China Phys. C 36, 573 (2012).

16. B.Q. Wang and X.H. Mo, China Phys. C 37, 026202 (2013). 